\title{
Incidencia de las características socioeconómicas en el rendimiento de los estudiantes - escala de lectura - de segundo grado de secundaria de la educación básica en la evaluación censal de estudiantes, según ámbito geográfico; Año 2015.
}

Incidence of socioeconomic characteristics in student performance - Reading scale - Second grade of secondary school of basic education in the Census Evaluation of Students, by geographical area; year 2015

Martín Efraín Mendoza Bolo

Escuela de Graduados. Pontificia Universidad Católica del Perú. Lima Perú.

\section{INFORMACIÓN}

Historia del Artículo

Recepción: 03/04/2019

Revisión: $12 / 05 / 2019$

Aceptación: 22/05/2019

\section{Palabras Clave}

Logro de aprendizaje, escala de Lectura, pobreza monetaria, índice socioeconómico, características socioeconómicas.

\section{Key Words}

learning achievement, Reading scale, monetary poverty, socioeconomic index, socioeconomic characteristics.

\section{DOI}

https://doi.org/10.35286/veritas. v20i2.239

\begin{abstract}
RESUMEN
Desde una aproximación cuantitativa, es factible identificar factores asociados al logro de aprendizaje de los estudiantes no solo desde la institución educativa. Hay que considerar el entorno del estudiante. $\mathrm{Y}$ en el entorno del estudiante, necesariamente, se identifica como un indicador el estrato socio-económico de la familia del estudiante. Las características socioeconómicas se van a describir, según ámbito geográfico, desde un índice socioeconómico (elaborado por la Unidad de Medición de la Calidad) y la Pobreza Monetaria (índice unidimensional recabado por el Instituto Nacional de Estadística e Informática) y se va a indagar por su relación con el rendimiento de los estudiantes de segundo grado de secundaria de la educación básica. De la metodología estadística utilizada se desprende que: (i) mayor porcentaje de pobreza monetaria de una región está asociada a un mayor porcentaje de estudiantes en el nivel de logro previo al inicio en la escala de lectura. (ii) mayor media ponderada de una región en la escala de lectura está asociada a un mayor índice socio económico. (iii) lo que prevalece, a nivel nacional, es la distancia - mensurable - entre el rendimiento escolar urbano y rural.
\end{abstract}

\begin{abstract}
From a quantitative approximation, it is feasible to identify factors associated with the learning achievement of students not only from the educational institution. The student's environment must be considered. And in the student's environment, necessarily, the socio-economic stratum of the student's family is identified as an indicator. Socioeconomic characteristics will be described, according to geographic scope, from a socioeconomic index (elaborated by the Quality Measurement Unit) and Monetary Poverty (one-dimensional index collected by the National Institute of Statistics and Informatics) and will be investigated for its relationship with the performance of students in the secondary grade of secondary basic education. From the statistical methodology used, it follows that: (i) the highest percentage of monetary poverty in a region is associated with a higher percentage of students in the level of achievement prior to the beginning of the Reading scale. (ii) greater weighted average of a region in the Reading scale is associated with a higher socioeconomic index. (iii) what prevails, at the national level, is the distance-measurable-between urban and rural school performance.
\end{abstract}

\section{INTRODUCCIÓN}

Para recabar información relacionada al rendimiento de los estudiantes de la educación básica, el Ministerio de Educación (Minedu) aplica a nivel nacional Evaluaciones Censales de Estudiantes (ECE). Sendas evaluaciones nos permiten discriminar niveles de logro de aprendizaje así como medir logros de aprendizaje satisfactorios de los estudiantes según ámbito geográfico. Los resultados obtenidos propician espacios de diálogo para aproximarnos a los procesos pedagógicos en el aula.

En la presente artículo nos proponemos realizar un

Correspondencia:

Martín Efraín Mendoza Bolo

mendozab.martin@pucp.edu.pe análisis descriptivo correlacional a partir de dos bases de datos: la data recabada por la Unidad de Medición de la Calidad Educativa (UMC) del Ministerio de Educación: Evaluación Censal de Estudiantes (ECE 2015), obtenida al aplicar el instrumento Escala de Lectura a los estudiantes de segundo grado de secundaria de la educación básica; y, la data de la Encuesta Nacional de Hogares 2015 (ENAHO) recabada por el Instituto Nacional de Estadística e Informática (INEI ). En particular, se va a indagar por las características socioeconómicas y su incidencia en el rendimiento de los estudiantes - escala de lectura - de segundo grado de secundaria de la educación básica en la Evaluación Censal de Estudiantes.

Desde el Ministerio de Educación, la Unidad de Medición de la Calidad Educativa (UMC) ha medido - a través de pruebas de opción múltiple y preguntas abiertas - logros de aprendizaje de los estudiantes a nivel nacional mediante Pruebas Nacionales: tercero y sexto grado de primaria y 
tercero y quinto grado de secundaria. La última evaluación nacional en el nivel secundario se realizó en el año 2004 (Evaluación Nacional 2004). Desde hace doce años, la UMC presenta los resultados generales de la Evaluación Censal de Estudiantes (ECE) de segundo grado de primaria. Estos resultados se reportan en base a la muestra de control de cada ECE. El año 2015 por primera vez se ha aplicado un cuestionario para el segundo grado de secundaria de la educación básica.

En el caso de evaluaciones internacionales los resultados en educación primaria como en secundaria - también - no son nada halagadores. Desde el Laboratorio Latinoamericano de Evaluación y Calidad Educativa (LLECE ) se aplican pruebas de medición de logros de aprendizaje para tercer y sexto grado de primaria, con el rótulo de Estudio Regional Comparativo y Explicativo (PERCE / SERCE / TERCE). Desde la OCDE se implementan las pruebas PISA para los países miembros así como para países invitados: se miden logros de aprendizaje en estudiantes de 15 años de edad (no se selecciona la muestra por grado de estudio).

Peña, S. (2005) precisa que "Desde la década del sesenta la economía de la educación -particularmente en la forma de teoría del capital humano- ha insistido en los vínculos, no del todo acreditados desde el punto de vista empírico, entre educación y desarrollo (no sabemos si los mayores niveles de educación son fruto del crecimiento o si es al revés), y la economía neoclásica ha insistido en el uso de incentivos y de subsidios a la demanda para mejorar la educación."

En esta línea Williams, D. (2006) al realizar un metanálisis acerca de los resultados en competencia lectora de las pruebas PIRLS (Progress in International Reading Literacy Study) del año 2001 y PISA (Programme for International Student Assessment) de los años 2000 y 2002; identifica que en la educación básica "Durante los años de la educación primaria y media, los niños tienen menos probabilidades de desempeñarse bien en las prácticas académicas o de participar en actividades escolares curriculares y extracurriculares si sus padres tienen ingresos bajos, un nivel de educación deficiente o están desempleados o tienen carreras de escaso prestigio".

Asimismo, Cueto et al (2008) al realizar un meta-análisis de tres estudios realizados acerca de las Oportunidades de Aprendizaje $\left(\mathrm{ODA}^{4}\right)$ en estudiantes de educación primaria (tercero, cuarto y sexto grado; áreas de matemática y comunicación) identificó que "Tanto en cobertura curricular como en retroalimentación se encontró una asociación con nivel socioeconómico de los estudiantes en el aula. Esto seguramente se explica porque los estudiantes de mayor nivel socioeconómico tienen mejor rendimiento al inicio del año escolar y por tanto pueden hacer más ejercicios durante el año (y aprender más al finalizar, agrandando la brecha de rendimiento que existía al inicio). Así, la escuela pública estaría dando más oportunidades de acuerdo a los antecedentes individuales y familiares de los estudiantes, desfavoreciendo el rol de la educación como un instrumento para la igualdad social."

\section{MATERIALES Y MÉTODOS}

La metodología de la investigación es cuantitativa; retrospectiva: descriptiva relacional no causal (ex-posfacto); bivariada.

Según la muestra ${ }^{5}$ de control de la ECE 2015, en la Escala de lectura (competencia lectora) solo el 14,72\% del total de estudiantes se encuentra en el Nivel Satisfactorio. Más de la mitad del total de estudiantes se encuentra en el Nivel en Inicio o Previo al Inicio $(62,66 \%=23,64 \%+$ $39,02 \%)$. Es decir, más del $60 \%$ de estudiantes o "Solo logra realizar tareas poco exigentes respecto de lo que se espera para el VI ciclo" o "no logró los aprendizajes necesarios para estar en el Nivel cn Inicio".

De la muestra de control, la cual es representativa a nivel regional, las regiones con mayor porcentaje de estudiantes en el Nivel Previo al Inicio (< Nivel 1) son Apurímac (50,38\%), Huancavelica (52,36\%), Huánuco (41,4\%), Loreto (44,66\%), Puno (39,08\%) y Amazonas (38,22\%).

Tabla 1: Desempeño escala de lectura. Fuente: elaboración propia.

\begin{tabular}{|c|c|c|c|c|c|}
\hline dptm & Nivel 1 & Nivel 1 & Nivel 2 & Nivel 3 & Total \\
\hline Amazonas & 38.22 & 39.08 & 15.68 & 7.02 & 100.00 \\
\hline Ancash & 32.19 & 38.48 & 18.68 & 10.65 & 100.00 \\
\hline Apurimac & 50.38 & 33.72 & 10.73 & 5.17 & 100.00 \\
\hline Arequipa & 9.12 & 34.25 & 31.38 & 25.24 & 100.00 \\
\hline Ayuacucho & 36.39 & 40.51 & 15.82 & 7.28 & 100.00 \\
\hline Cajamarca & 34.48 & 43.05 & 15.35 & 7.12 & 100.00 \\
\hline Callao & 9.67 & 37.54 & 31.58 & 21.20 & 100.00 \\
\hline Cusco & 35.46 & 36.70 & 17.41 & 10.43 & 100.00 \\
\hline Huancavelica & 52.36 & 35.49 & 8.80 & 3.35 & 100.00 \\
\hline Huánuco & 41.40 & 39.29 & 13.41 & 5.90 & 100.00 \\
\hline Ica & 14.92 & 41.75 & 26.81 & 16.53 & 100.00 \\
\hline Junín & 21.90 & 41.52 & 23.25 & 13.33 & 100.00 \\
\hline La Libertad & 22.33 & 40.33 & 22.60 & 14.74 & 100.00 \\
\hline Lambayeque & 19.98 & 43.42 & 22.78 & 13.82 & 100.00 \\
\hline Lima & 11.26 & 36.50 & 29.49 & 22.75 & 100.00 \\
\hline Loreto & 44.66 & 39.14 & 11.67 & 4.53 & 100.00 \\
\hline Madre de Dios & 26.15 & 45.81 & 20.41 & 7.63 & 100.00 \\
\hline Moquegua & 8.59 & 33.20 & 31.19 & 27.02 & 100.00 \\
\hline Pasco & 27.01 & 41.18 & 21.23 & 10.59 & 100.00 \\
\hline Piura & 22.63 & 43.52 & 21.64 & 12.22 & 100.00 \\
\hline Puno & 39.08 & 38.94 & 15.16 & 6.82 & 100.00 \\
\hline San Martín & 29.92 & 46.38 & 16.72 & 6.98 & 100.00 \\
\hline Tacna & 8.15 & 32.27 & 33.50 & 26.07 & 100.00 \\
\hline Tumbes & 25.36 & 46.06 & 20.27 & 8.30 & 100.00 \\
\hline Ucayali & 33.06 & 43.77 & 16.50 & 6.68 & 100.00 \\
\hline Total & 23.64 & 39.02 & 22.61 & 14.72 & 100.00 \\
\hline
\end{tabular}




\section{Variables independientes}

- $\mathrm{X}_{1}$ : índice socioeconómico $\left(\right.$ ise $\left.^{6}\right)$ por región.

- $\mathrm{X}_{2}$ : proporción de personas pobres monetarias por región (según INEI).

\section{Variables dependientes}

- $Y_{1}$ : puntaciones promedio ponderadas por ámbito geográfico definido.

- $Y_{2}$ : porcentaje de estudiantes con nivel de logro previo al inicio $(<$ Nivel 1) por región.

Cabe explicitar que la asociación lineal se puede describir mediante un gráfico de dispersión y mediante una ecuación lineal entre la proporción de estudiantes en un determinado nivel de logro de aprendizaje versus la proporción de personas pobres monetarias.

Para fines descriptivos del presente artículo, se han formulado la siguiente clasificación, según ámbitos geográficos:

- Regiones Naturales: Costa (Callao, Ica, La Libertad, Lambayeque, Lima ${ }^{7}$, Moquegua, Piura, Tacna, Tumbes); Sierra (Ancash, Apurímac, Arequipa ${ }^{8}$ Ayacucho, Cajamarca, Cusco, Huancavelica, Huánuco, Junín, Pasco, Puno) y Selva (Amazonas, Loreto, Madre de Dios, San Martín, Ucayali)

- Dominios: Costa urbano; Costa rural; Sierra urbano; Sierra rural; Selva urbano y Selva rural. Un Dominio es la intersección de la variable "región natural" y la variable "Area" (etiqueta: Area9).

- Macro regiones: Capital (Lima y Callao); Centro (Apurímac; Ayacucho, Huancavelica, Huánuco, Junín, Pasco.); Sur (Arequipa, Cusco, Ica, Moquegua, Puno, Tacna); Norte (Áncash, Cajamarca, La Libertad, Lambayeque, Piura, Tumbes) y Oriente $^{10}$ (Amazonas, Loreto, Madre de Dios, San Martín, Ucayali).

- Macro áreas: Capital urbano; Capital rural; Centro urbano; Centro rural; Sur urbano; Sur rural; Norte urbano; Norte rural; Oriente urbano; Oriente rural. Una Macro área es la intersección de la variable "Macro Región" y la variable "Área" (etiqueta: Area).

\section{RESULTADOS}

3.1 Escala de lectura: puntuaciones promedio ponderadas $\left(\mathrm{Y}_{1}\right)$ versus Índice Socio Económico $\left(\mathrm{X}_{1}\right)$

Sin pérdida de generalidad se pueden bosquejar diagramas de dispersión (scatter) que expliciten algún tipo de relación entre las puntuaciones promedio ponderadas y el Índice Socio Económico (Ise).

Al compilar las medias por región natural obtenemos: Costa $(579,68)$, Sierra $(544,27)$ y Selva $(530,94)$. Sabiendo que el promedio ponderado nacional en las puntuaciones de Comprensión Lectora es 561,5 y la desviación estándar es 73,66; según región natural, se identifica que el promedio de la Costa es mayor que el de la Sierra en 0,481 desviaciones estándar y respecto de la Selva es 0,662 desviaciones estándar. La única región natural sobre el promedio ponderado es la Costa.

Al compilar las medias por Dominios obtenemos: Costa urbano $(582,49)$; Costa rural $(518,27)$; Sierra urbano $(555,49)$; Sierra rural $(498,56)$; Selva urbano $(539,39)$ y Selva rural $(492,97)$. Según Dominios, sabiendo que la puntuación media de la región natural Costa es 579,68; se observa que en la diferencia entre Costa urbano y rural es 0,872 desviaciones estándar. El promedio de la región natural Sierra es 544,27 y la diferencia entre Sierra urbano y rural es 0,773 desviaciones estándar. Además, la puntuación media de la región natural Selva es 530,94 y la diferencia entre Selva urbano y rural es 0,63 desviaciones estándar. El único Dominio sobre el promedio ponderado es la Costa urbano.

Al determinar un diagrama de dispersión entre la media por Dominios y el Ise, se obtiene:

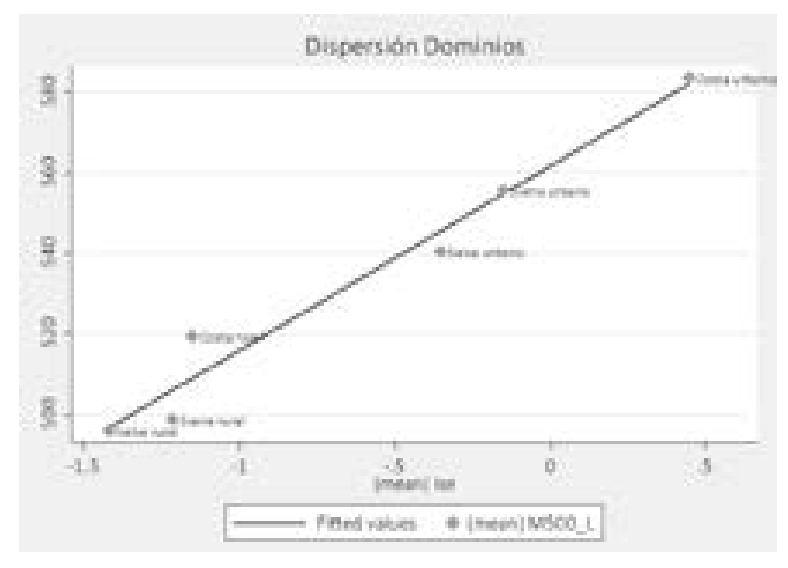

Fig. 1: Dispersión Ise vs Media por Dominios. Fuente: elaboración propia.

Obsérvese que en el extremo superior derecho se identifica a la Costa urbano y en el extremo inferior izquierdo se identifica la selva rural. Asimismo, es fácil corroborar las distancias (expresadas en desviaciones estándar) entre los elementos del Dominio. Se identifica predominio de lo urbano sobre lo rural.

Al compilar las medias por Macro regiones obtenemos: $\operatorname{Kap}^{11}(588,91)$, Centro $(537,06)$, Sur $(561,19)$, Norte $(553,63)$ y Oriente $(530,94)$.

Conociendo que el promedio nacional en las puntuaciones de Comprensión Lectora es 561,5; la única macro región sobre el promedio es la Capital (Kap). La diferencia entre la macro región capital y la macro región oriente es 0,67 desviaciones estándar.

Al determinar un diagrama de dispersión entre la media por Macro regiones y el Ise, se obtiene:

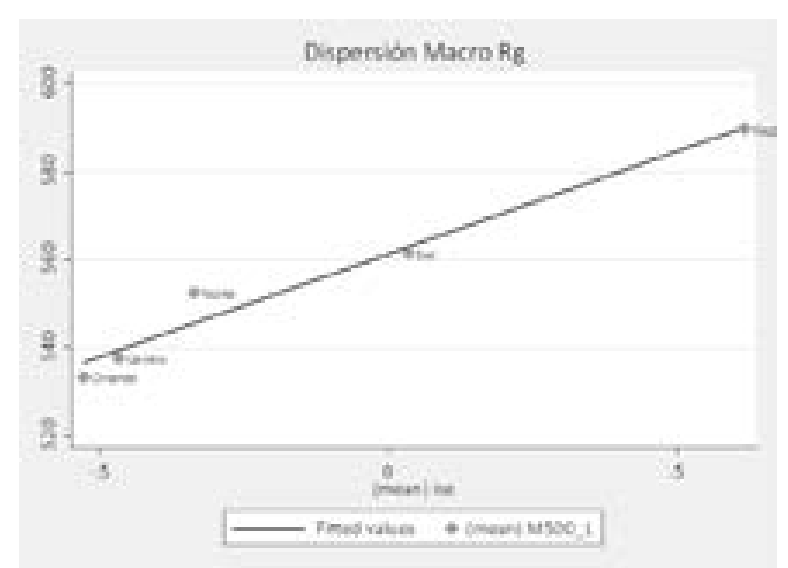

Fig. 2: Dispersión Ise vs Media por Macro regiones. Fuente: elaboración propia. 
Obsérvese que en el extremo superior derecho se identifica la Macro región capital y en el extremo inferior izquierdo se identifica la Macro región Oriente. Próximo al promedio converge la Macro región Sur.

Al compilar las medias por Macro área obtenemos: Capital urbano $(589,28)$, Capital rural $(555,67)$, Centro urbano $(547,31)$, Centro rural $(496,29)$, Sur urbano $(569,09)$, Sur rural $(499,74)$, Norte urbano $(563,55)$, Norte rural $(505,25)$, Oriente urbano $(539,39)$ y Oriente rural $(492,97)$.

Las Macro áreas sobre el promedio nacional son: Capital urbano (kap_ub), Sur urbano (sur_ub) y Norte urbano (nrt_ub). El Oriente urbano (ort_ub) está por debajo de la media nacional. En particular, se observa que la diferencia entre Capital urbano y Oriente urbano es 0,677 desviaciones estándar; y, la diferencia entre Capital rural (kap_rr) y Oriente rural (ort rr) es 0,851 desviaciones estándar.

Al determinar un diagrama de dispersión entre la media por Macro áreas y el Ise, se obtiene:

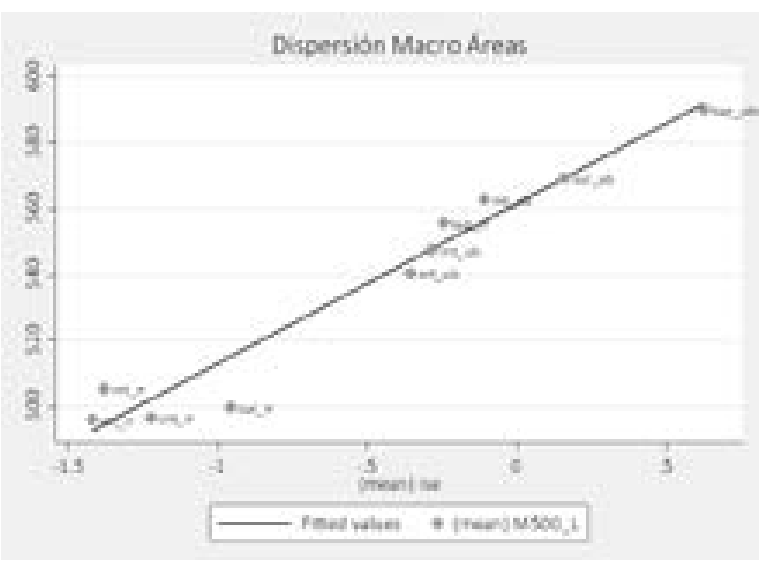

Fig. 3: Dispersión Ise vs Media por Macro áreas. Fuente: elaboración propia.

Obsérvese que en el extremo superior derecho se identifica la Macro área Capital urbano (kap_ub) y en el extremo inferior izquierdo se identifican las Macro áreas: Oriente rural (ort_rr), Norte rural (nrt_rr), Centro rural (cnt rr) y Sur rural (sur_rr). Se identifica predominio de lo urbano sobre lo rural.

En particular las Macro áreas Oriente rural, Norte rural y Centro rural tienen un Ise (coeficiente) entre $-1,5$ y -1 . Además, la Macro área Capital rural tiene un promedio ponderado mayor que Centro urbano y Oriente urbano.

Finalmente, al elaborar un diagrama de dispersión entre el puntaje promedio ponderado por región ${ }^{12}\left(\mathrm{Y}_{1}\right)$ versus el Ise $\left(\mathrm{X}_{1}\right)$, se identifica una correlación directa (positiva):

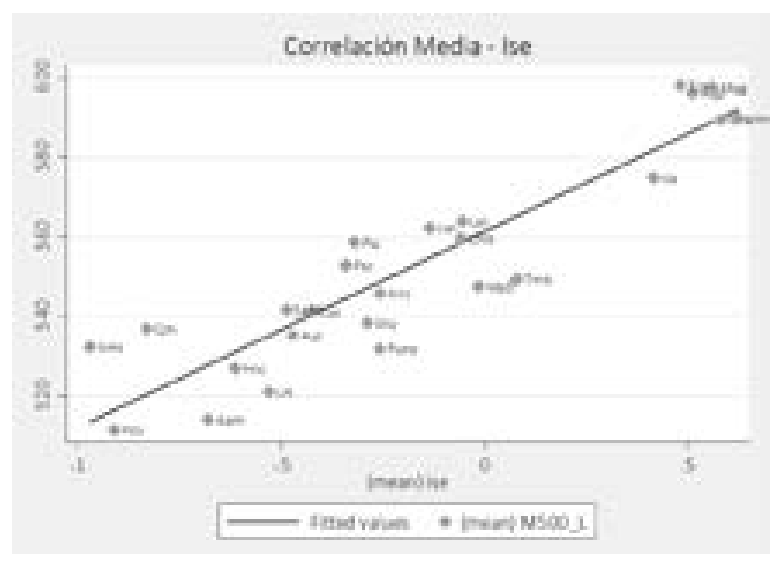

Fig. 4: Dispersión Ise vs media ponderada por región. Fuente: elaboración propia.

Como se observa en el gráfico adjunto, las puntuaciones promedios más altas (media regional ponderada) son las de Tacna $(597,95)$, Moquegua $(597,35)$ y Arequipa $(595,8)$. Según el gráfico adjunto, las puntuaciones promedio por región próximas a 600 tienen mayor coeficiente Ise (características socioeconómicas). Sin embargo, es fácil ver que Lima $(588,94)$ y Callao $(588,64)$ tienen mayor coeficiente Ise que Tacna, Arequipa y Moquegua pero su puntuación promedio es menor. Tener en cuenta que el promedio nacional en las puntuaciones de Comprensión Lectora es 561,5.

Escala de lectura y niveles de logro de aprendizaje, según ámbito geográfico

Según ámbito geográfico, la región natural Costa tiene el 19,48\% de estudiantes en el Nivel Satisfactorio, la región natural sierra tiene el $10,38 \%$ de estudiantes en este nivel y la región natural Selva solo tiene el $6,14 \%$ de estudiantes en este nivel. El ratio [(Nivel 3) / $(<$ Nivel 1)], según región natural, para la Costa es [1,33], para la Sierra es $[0,32]$ y para la Selva es $[0,17]$. Salvo la Costa, los otros ratios son menores que 1.

Según Dominios, la Costa urbano tiene el 20,2\% de estudiantes en el Nivel Satisfactorio (Nivel 3). La menor proporción de estudiantes en el Nivel Satisfactorio lo tienen la Costa rural, Sierra rural y Selva rural. La menor proporción de estudiantes en el Nivel Satisfactorio y la mayor proporción de estudiantes en el Nivel Previo al Inicio, lo tiene la Selva rural:

Tabla 2: Desempeño escala de lectura, según dominio. Fuente: elaboración propia.

\begin{tabular}{cccccc}
\hline Dom_rg & $<$ Nivel 1 & Nivel 1 & Nivel 2 & Nivel 3 & Total \\
\hline Costa urbano & 13.25 & 38.53 & 28.02 & 20.20 & 100.00 \\
Costa rural & 45.71 & 39.99 & 10.63 & 3.67 & 100.00 \\
Sierra urbano & 26.07 & 40.27 & 21.12 & 12.54 & 100.00 \\
Sierra rural & 59.70 & 32.61 & 6.13 & 1.57 & 100.00 \\
Selva urbano & 31.29 & 44.55 & 16.96 & 7.21 & 100.00 \\
Selva rural & 60.31 & 32.24 & 6.12 & 1.34 & 100.00 \\
Total & 23.64 & 39.02 & 22.61 & 14.72 & 100.00 \\
\hline
\end{tabular}


Además, según Dominios en el Nivel Satisfactorio el ratio [urbano/ rural] en la costa es [5,5]; en la Sierra es [7,99] $y$ en la Selva es [5,38].

Según Macro regiones, la Capital tiene el 22,61\% de estudiantes en el Nivel Satisfactorio (Nivel 3). EL Oriente solo tiene el $6,14 \%$ de estudiantes en este nivel. Asimismo, más del $60 \%$ de estudiantes se encuentra en el Nivel en Inicio o Previo al Inicio en las Macro regiones Centro $(75,17 \%)$, Sur $(61,95 \%)$, Norte $(68,19 \%)$ y Oriente $(78,88 \%)$ :

Tabla 3: Desempeño escala de lectura, según Macro regiones. Fuente: elaboración propia.

\begin{tabular}{cccccc}
\hline mc_rg & $<$ Nivel 1 & Nivel 1 & Nivel 2 & Nivel 3 & Total \\
\hline Kap & 11.11 & 36.60 & 29.68 & 22.61 & 100.00 \\
Centro & 35.99 & 39.18 & 16.48 & 8.34 & 100.00 \\
Sur & 24.91 & 37.04 & 22.79 & 15.26 & 100.00 \\
Norte & 26.19 & 42.00 & 20.22 & 11.60 & 100.00 \\
Oriente & 36.57 & 42.31 & 14.98 & 6.14 & 100.00 \\
Total & 23.64 & 39.02 & 22.61 & 14.72 & 100.00 \\
\hline
\end{tabular}

El ratio [(Nivel 3) / (< Nivel 1)], según Macro regiones, para la Capital es [2,04], para el Centro es [0,23], para el Sur es $[0,61]$, para el Norte es $[0,44]$ y para el Oriente es $[0,17]$. Salvo la Capital, los otros ratios son menores que 1.

Según Macro áreas, la Capital urbano tiene el 22,75\% de estudiantes en el Nivel Satisfactorio (Nivel 3). La menor proporción de estudiantes en el Nivel Satisfactorio lo tienen el Centro rural, Sur rural, Norte rural y Oriente rural. Más del $60 \%$ de estudiantes del Centro rural y Oriente rural se encuentran en el Nivel Previo al Inicio:

Tabla 4: Desempeño escala de lectura, según Macro áreas. Fuente: elaboración propia.

\begin{tabular}{cccccc}
\hline areag & $<$ Nivel 1 & Nivel 1 & Nivel 2 & Nivel 3 & Total \\
\hline kap_ub & 10.99 & 36.49 & 29.76 & 22.75 & 100.00 \\
kap_rr & 22.28 & 45.85 & 21.99 & 9.87 & 100.00 \\
cnt_ub & 29.60 & 41.17 & 19.18 & 10.06 & 100.00 \\
cnt_rr & 61.45 & 31.29 & 5.78 & 1.49 & 100.00 \\
sur_ub & 20.41 & 37.85 & 24.82 & 16.91 & 100.00 \\
sur_rr & 59.89 & 30.70 & 7.00 & 2.41 & 100.00 \\
nrt_ub & 20.46 & 42.99 & 22.92 & 13.63 & 100.00 \\
nrt_rr & 54.15 & 37.14 & 7.04 & 1.67 & 100.00 \\
ort_ub & 31.29 & 44.55 & 16.96 & 7.21 & 100.00 \\
ort_rr & 60.31 & 32.24 & 6.12 & 1.34 & 100.00 \\
Total & 23.64 & 39.02 & 22.61 & 14.72 & 100.00 \\
\hline
\end{tabular}

Además, según Macro áreas, en el Nivel Satisfactorio el ratio [urbano/rural] en la Capital es [2,3], en el Centro es $[6,75]$, en el Sur es $[7,02]$, en el Norte es $[8,16]$ y en el Oriente es $[5,38]$.

\section{Pobreza monetaria, según ámbito geográfico}

Como metodología la Pobreza Monetaria es unidimensional. En nuestro país se mide la capacidad de gasto per cápita del hogar. Para el año 2015, según el INEI, se tiene que las regiones con mayor pobreza extrema son Cajamarca (20,26\%), Ayacucho (10,63\%), Huancavelica (10,5\%) y Amazonas (10,5\%). Las regiones con mayor proporción de pobres no extremos son Huancavelica (34,65\%), Amazonas (31,79\%), Apurímac (31,14\%), Pasco (31,05\%), Cajamarca (30,59\%) y Ayacucho (30,1\%). Para motivos del presente artículo la distinción entre pobre extremo y pobre no extremo monetario es prescindible dado que nos interesa identificar la proporción de pobres monetarios por ámbito geográfico. En consecuencia, se puede formular el siguiente cuadro:

Tabla 5: Pobreza monetaria por región. Fuente: elaboración propia.

\begin{tabular}{|c|c|c|c|}
\hline dptm & No PobreM & PobreM & Total \\
\hline Amazonas & 57.71 & 42.29 & 100.00 \\
\hline Ancash & 75.95 & 24.05 & 100.00 \\
\hline Apurimac & 61.34 & 38.66 & 100.00 \\
\hline Arequipa & 91.76 & 8.24 & 100.00 \\
\hline Ayuacucho & 59.28 & 40.72 & 100.00 \\
\hline Cajamarca & 49.15 & 50.85 & 100.00 \\
\hline Callao & 88.46 & 11.54 & 100.00 \\
\hline Cusco & 82.40 & 17.60 & 100.00 \\
\hline Huancavelica & 54.84 & 45.16 & 100.00 \\
\hline Huánuco & 64.40 & 35.60 & 100.00 \\
\hline Ica & 95.04 & 4.96 & 100.00 \\
\hline Junín & 80.90 & 19.10 & 100.00 \\
\hline La Libertad & 74.14 & 25.86 & 100.00 \\
\hline Lambayeque & 79.24 & 20.76 & 100.00 \\
\hline Lima & 88.50 & 11.50 & 100.00 \\
\hline Loreto & 65.03 & 34.97 & 100.00 \\
\hline Madre de Dios & 92.85 & 7.15 & 100.00 \\
\hline Moquegua & 92.23 & 7.77 & 100.00 \\
\hline Pasco & 61.47 & 38.53 & 100.00 \\
\hline Piura & 70.57 & 29.43 & 100.00 \\
\hline Puno & 65.36 & 34.64 & 100.00 \\
\hline San Martín & 72.45 & 27.55 & 100.00 \\
\hline Tacna & 84.87 & 15.13 & 100.00 \\
\hline Tumbes & 87.02 & 12.98 & 100.00 \\
\hline Ucayali & 88.56 & 11.44 & 100.00 \\
\hline Total & 78.23 & 21.77 & 100.00 \\
\hline
\end{tabular}

Obsérvese que la proporción total $(78,23 \%)$ de no pobres se mantiene. La pobreza monetaria para el año 2015 es $21,77 \%$ (proporción de pobres nacional). Las regiones con mayor pobreza monetaria son Cajamarca $(50,85 \%)$, Huancavelica (45,16\%), Amazonas (42,29\%) y Ayacucho $(40,72 \%)$. Las regiones con menor pobreza monetaria son Ica $(4,96 \%)$, Madre de Dios (7,15\%), Moquegua $(7,77 \%)$ y Arequipa $(8,24 \%)$. 
Según ámbito geográfico ${ }^{13}$, la región natural Costa tiene el $15,38 \%$ de pobreza monetaria, la Sierra tiene el $29,95 \%$ de pobreza monetaria y la selva tiene el $28,66 \%$ de pobreza monetaria.

Según Dominios, la pobreza monetaria es mayor en el área rural en las tres regiones naturales:

Tabla 6: Pobreza Monetaria según Dominio. Fuente: elaboración propia.

\begin{tabular}{cccc}
\hline Dom_rg & No PobreM & PobreM & Total \\
\hline Costa urbano & 87.04 & 12.96 & 100.00 \\
Costa rural & 57.04 & 42.96 & 100.00 \\
Sierra urbano & 83.61 & 16.39 & 100.00 \\
Sierra rural & 53.59 & 46.41 & 100.00 \\
Selva urbano & 78.31 & 21.69 & 100.00 \\
Selva rural & 57.77 & 42.23 & 100.00 \\
Total & 78.23 & 21.77 & 100.00 \\
\hline
\end{tabular}

El ratio [rural/urbano] en la Costa es [3,315], en la Sierra es $[2,832]$ y en la Selva es $[1,947]$.

Según Macro regiones, la mayor proporción de pobres monetarios se encuentra en la Macro región Centro (32,81\%). La menor proporción de pobres monetarios se encuentra en la Macro región Capital (11,51\%).

Según Macro áreas, la pobreza monetaria es mayor en el área rural. El ratio [rural/urbano] en la Capital es [1,54], en el Centro es [2,2], en la Sur es [3,17], en el Norte es [2,99] y en el Oriente es $[1,95]$.

Tabla 7: Pobreza Monetaria según Macro áreas. Fuente: elaboración propia.

\begin{tabular}{cccc}
\hline areag & No PobreM & PobreM & Total \\
\hline Kap_ub & 88.60 & 11.40 & 100.00 \\
Kap_rr & 82.42 & 17.58 & 100.00 \\
Cnt_ub & 79.49 & 20.51 & 100.00 \\
Cnt_rr & 54.83 & 45.17 & 100.00 \\
Sur_ub & 89.10 & 10.90 & 100.00 \\
Sur_rr & 65.48 & 34.52 & 100.00 \\
Nrt_ub & 81.60 & 18.40 & 100.00 \\
Nrt_rr & 45.04 & 54.96 & 100.00 \\
Ort_ub & 78.31 & 21.69 & 100.00 \\
Ort_rr & 57.77 & 42.23 & 100.00 \\
Total & 78.23 & 21.77 & 100.00 \\
\hline
\end{tabular}

3.3.1 Coeficiente de Pearson y diagrama de dispersión de las puntuaciones promedio ponderadas por región (Y1) de los estudiantes de segundo grado en la Escala de Lectura versus proporción de personas pobres monetarias según ámbito regional $(\mathrm{X} 2)$.

El coeficiente de Pearson es -0,7256. El diagrama de dispersión con un intervalo de confianza al 95\% se presenta a continuación:

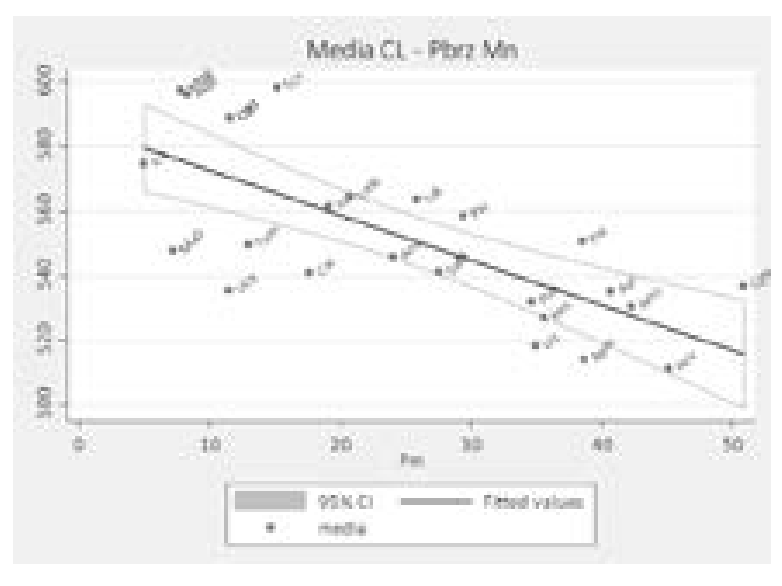

Fig. 5: Dispersión Pobreza Monetaria vs Media ponderada por Región.

Del diagrama de dispersión se puede afirmar, sin pérdida de generalidad, que a mayor proporción de personas pobres monetarias se le asocia una menor puntuación promedio ponderada por región.

Logros de aprendizaje en la Escala de Lectura ( $\left.\mathrm{Y}_{2}\right)$ versus Pobreza Monetaria $\left(\mathrm{X}_{2}\right)$; donde Y2: porcentaje de estudiantes con nivel de logro previo al inicio por región ( $\mathrm{Y}_{2}=\mathrm{Lg} \_$inf)

Al efectuar la regresión de Y2 con X2 obtenemos:

Tabla 8: Regresión Logro previo al Inicio vs. Pobreza Monetaria por región. Fuente: elaboración propia.

\begin{tabular}{|c|c|c|c|c|c|c|}
\hline Source & SS & $\mathrm{df}$ & & & \multirow{4}{*}{\multicolumn{2}{|c|}{$\begin{array}{c}\text { Number of obs }=25 \\
F(1,23)=30.36 \\
\text { Prob }>F=0.0000 \\
\text { R-squared }=0.5690 \\
\text { Adj R-squared }=0.5502 \\
\text { Root MSE }=8.7452\end{array}$}} \\
\hline Model & \multirow{2}{*}{$\begin{array}{l}2321.86995 \\
1759.00175\end{array}$} & 1 & \multicolumn{2}{|c|}{2321.86995} & & \\
\hline Residual & & 23 & \multicolumn{2}{|c|}{76.478337} & & \\
\hline Total & 4080.87 .17 & 24 & \multicolumn{2}{|c|}{170.036321} & & \\
\hline Lg_inf & Coef. & Std. Err. & $\mathrm{t}$ & $\mathrm{p}>|\mathrm{t}|$ & {$[95 \%$ Con } & Interval] \\
\hline $\mathrm{Pm}$ & .7171442 & .1301538 & 5.51 & 0.000 & .4479006 & .9863878 \\
\hline _cons & 10.102 .92 & 3.655126 & 2.76 & 0.011 & 2.541713 & 17.66412 \\
\hline
\end{tabular}

Del resultado obtenido, identificamos que la ecuación de la recta de regresión es: $\mathrm{Y}_{2}=10,1+0,72\left(X_{2}\right)$

Dado el modelo lineal, la ordenada en el origen nos dice que cuando la pobreza monetaria es cero (ausencia de pobreza), el porcentaje de estudiantes con nivel de logro previo al inicio sería $10,1 \%$. Asimismo, al incrementar en una unidad (punto porcentual) la pobreza monetaria, la ordenada $\mathrm{Y}_{2}$ se incrementa en 0,72 unidades.

El coeficiente de Pearson es 0,7543. Lo cual se puede verificar a partir del valor de $\mathrm{R}^{2}(\sqrt{ } 0,569=0,7543)$. Se puede afirmar que $56,9 \%$ es el porcentaje de varianza común entre Y2 y X2. Recordar que cuanto mayor es el valor de $\mathrm{R}^{2}$, el error del modelo ${ }^{14}$ será menor.

Dado que Prob $>\mathrm{F}=0,0000$ (p-valor $<0,05$ ), la relación entre $\mathrm{Y}_{2}$ y $\mathrm{X}_{2}$ es estadísticamente significativa. En consecuencia, se identifica una asociación lineal directa entre el porcentaje de estudiantes con nivel de logro previo al inicio por región y la proporción de personas pobres monetarias. Es decir, mayor proporción de personas pobres monetarias de una región se relaciona linealmente con mayor porcentaje de estudiantes con nivel de logro previo al inicio. 
El diagrama de dispersión con un intervalo de confianza al $95 \%$ se presenta a continuación:

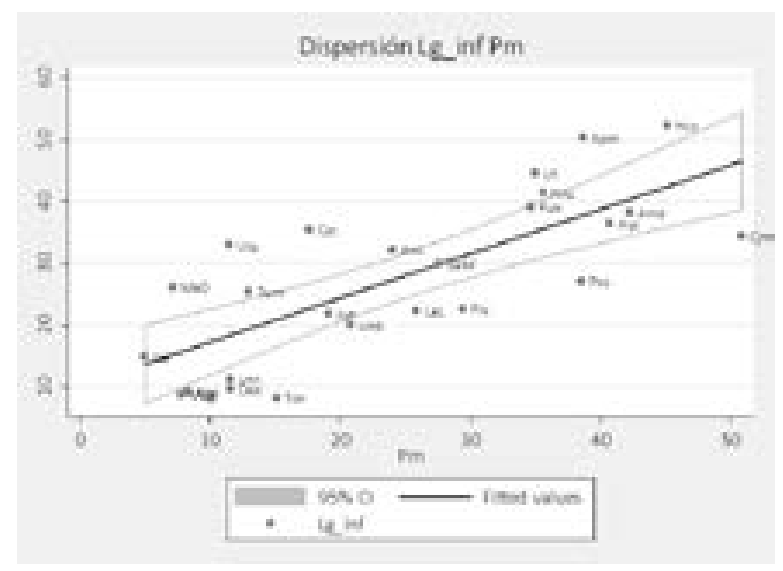

Fig. 6: Dispersión Pobreza Monetaria vs Logro previo al Inicio por región. Fuente: elaboración propia.

\section{DISCUSIÓN}

Según la muestra de control, del total de estudiantes el $88,71 \%$ pertenece al área geográfica urbana y el $11,29 \%$ pertenece al área geográfica rural. Según región natural, se tiene que la procedencia de los estudiantes en las tres regiones es mayoritariamente de gestión estatal ${ }^{15}$ : Costa $(64,63 \%)$, Sierra $(84,58 \%)$ y Selva $(94,41)$.

Teniendo en cuenta la data obtenida se identifica que:

- Según Dominio y según Macro área, la proporción de estudiantes en el Nivel Previo al Inicio es mayor en el área geográfica rural que en la urbana.

- Según Dominio y según Macro área, la proporción de pobres monetarios es mayor en el área geográfica rural que en la urbana.

Según la muestra de control de la ECE-2015 se puede discriminar por niveles de logro de aprendizaje el rendimiento de los estudiantes así como de las puntuaciones promedio ponderadas, según ámbito regional. Asimismo, desde la base de datos de la ENAHO 2015, se ha identificado la proporción de pobres monetarios según ámbito regional. En consecuencia, se puede afirmar que:

- Mayor porcentaje de pobreza monetaria de una región está asociada a un mayor porcentaje de estudiantes en el nivel de logro previo al inicio en la escala de Lectura.

- Mayor media ponderada de una región en la escala de lectura está asociada a un mayor índice socio económico. - Lo que prevalece, a nivel nacional, es la distancia mensurable - entre el rendimiento escolar urbano y rural.

\section{REFERENCIAS BIBLIOGRÁFICAS}

1. https://www.inei.gob.pe/bases-de-datos/

2. http://umc.minedu.gob.pe/evaluacion-censal-deestudiantes-2006-ece-2006/

3. http://www.llece.org/public/

4. Según Cueto et al "El concepto alude principalmente al nivel en el cual los estudiantes han sido expuestos en el aula a los temas que se incluyen en la evaluación. Así, las oportunidades de aprendizaje deberían tener un poder explicativo significativo del rendimiento (a mayores ODA, mayor rendimiento)."
5. http://umc.minedu.gob.pe/wp-content/ uploads/2016/07/Reporte-Tecnico-ECE-2015.pdf

6. Información en detalle sobre el diseño, formulación, aplicación y validación del ise se puede encontrar en: http://umc.minedu.gob.pe/desafios-en-la-mediciony-el-analisis-del-estatus-socioeconomico-de-losestudiantes-peruanos/

7. "Lima = Lima Metropolitana + Lima Provincias"

8. Cada región se toma como una unidad. Es fácil ver que la región Arequipa tiene tres provincias (Caravelí, Camaná e Islay) en la costa pero se considera como "un elemento" del conjunto "región natural Sierra".

9. Según la base de datos de la UMC, la variable "Area" discrimina entre instituciones educativas ubicadas en zona "urbano" e instituciones educativas ubicadas en zona "rural".

10. Obsérvese que la región natural Selva coincide con la Macro región Oriente.

11. Kap := Macro región Capital (Lima y Callao)

12. Amazonas $=\mathrm{Amz} / \mathrm{Ancash}=\mathrm{Anc} /$ Apurímac $=\mathrm{Apm} /$ Arequipa $=$ Aqp $/$ Ayacucho $=$ Ayc $/$ Cajamarca $=$ Cjm / $\mathrm{Callao}=\mathrm{Cao} \quad / \mathrm{Cusco}=\mathrm{Cus} /$ Huancavelica $=\mathrm{Hcv}$ / Huánuco $=\mathrm{Hnc} / \mathrm{Ica}=\mathrm{Ica} / \mathrm{Junín}=\mathrm{Jun} / \mathrm{La}$ Libertad $=\mathrm{LaL}$ $/$ Lambayeque $=\mathrm{Lmb} /$ Lima $=$ Lima $/$ Loreto $=$ Lrt / Madre de Dios $=\mathrm{MaD} /$ Moquegua $=\mathrm{Mqg} / \mathrm{Pasco}=\mathrm{Psc} /$ Piura $=$ Piu $/$ Puno $=$ Puno $/$ San Martín=SaM $/$ Tacna $=$ Tcn $/$ Tumbes $=\mathrm{Tmb} /$ Ucayali $=$ Ucy.

13. Cabe precisar que para fines de la presente investigación, se ha tomado como referencia inicial la base de datos de la ECE-2015 de la UMC. Contrastando con la base de datos del INEI, es fácil ver que la desagregación por ámbito geográfico del INEI es más fina. Por ejemplo, si bien es cierto que se considera la región Amazonas como Selva, según nuestro desagregado SIEMPRE será Selva. Sin embargo, según desagregado del INEI la región Amazonas como un todo tiene un subconjunto que pertenece a la Sierra. Lo mismo se puede verificar con otras regiones: es el caso de Ancash que tiene "parte de Costa".

14. $\mathrm{R}^{2}$ es el coeficiente de determinación; el cual mide la "bondad del ajuste" del modelo de regresión lineal.

15. Ratio nacional aprox: $75 \%$ estatal; $25 \%$ no estatal.

16. CUETO, Santiago y otros 2008 "Oportunidades de aprendizaje y rendimiento escolar en matemática y lenguaje: resumen de tres estudios en Perú". REICE - Revista Electrónica Iberoamericana sobre Calidad, Eficacia y Cambio en Educación, Vol. 6, No.1

17. CUETO, Santiago 2007 "Las Evaluaciones Nacionales e Internacionales de Rendimiento Escolar en el Perú: Balance y Perspectivas" en "Investigación, políticas y desarrollo en el Perú". GRADE, Lima.

18. GANIMIAN, Alejandro 2015 "Bajos resultados, altas mejoras ¿Cómo les fue a los estudiantes peruanos de primaria y secundaria en las últimas evaluaciones internacionales?" Oficina de Medición de la Calidad de los Aprendizajes. MINEDU, Lima.

19. MINISTERIO DE EDUCACIÓN

20. Minedu 2015a "Marco de Fundamentación de las Pruebas de Rendimiento de la Evaluación Censal de Estudiantes de $2^{\circ}$ de Secundaria”. UMC, Lima.

21. 2015b "Reporte Técnico de la Evaluación Censal de Estudiantes (ECE 2015)". UMC, Lima. 
22. 2013 "PISA 2012: Primeros Resultados" Informe Nacional del Perú". UMC, Lima.

23. 2009 Diseño Curricular Nacional de la Educación Básica Regular. DGEBR - DES, Lima.

24. 2005 EVALUACIÓN NACIONAL 2004: Marco de Trabajo de los Instrumentos de Factores Asociados al Rendimiento Estudiantil. Unidad de Medición de la Calidad Educativa, Lima.

25. OCDE - PISA 2016 "Estudiantes de bajo rendimiento. Por qué se quedan atrás y cómo ayudarles a tener éxito" Resumen Ejecutivo. Programa para la Evaluación Internacional de Alumnos, París.

26. PEÑA, Saúl 2005 "Igualdad educativa y sociedad democrática" en "Políticas educativas y equidad" UNICEF, Santiago de Chile.

27. WILLIAMS, Douglas 2006 "Las brechas de Aprendizaje: diez preguntas de la política educativa a seguir en relación con el desempeño y la equidad en las escuelas y los sistemas educativos". Instituto de Estadística de la UNESCO, Montreal.

28. "Desafíos en la medición y el análisis del estatus socioeconómico de los estudiantes peruanos". http:// umc.minedu.gob.pe/desafios-en-la-medicion-yel-analisis-del-estatus-socioeconomico-de-losestudiantes-peruanos/

29. Informe para la Institución Educativa ¿Qué logran nuestros estudiantes en la ECE? ECE $20152^{\circ}$ grado de secundaria / UMC http://umc.minedu. gob.pe/wp-content/uploads/2016/03/Informe-paraInstituci\%C3\%B3n-Educativa-sec_ECE-2015.pdf

30. "Marco de Fundamentación de las Pruebas de Rendimiento de la Evaluación Censal de Estudiantes de $2^{\circ}$ de Secundaria" / UMC http://umc.minedu.gob. pe/wp-content/uploads/2015/08/Marco-de-la-ECE$2 \%$ C2\%BA.-de-secundaria.pdf

31. "Reporte Técnico de la Evaluación Censal de Estudiantes (ECE 2015)"/UMC http://umc.minedu. gob.pe/wp-content/uploads/2016/07/Reporte-TecnicoECE-2015.pdf 